\title{
Empirical Bayesian Mixture Models for Medical Image Translation
}

\author{
Mikael Brudfors ${ }^{1}$, John Ashburner ${ }^{1}$, Parashkev Nachev $^{2}$, and Yaël Balbastre ${ }^{1}$ \\ 1 Wellcome Centre for Human Neuroimaging, UCL, London, UK \\ \{mikael.brudfors.15,j.ashburner, y. balbastre\} @ucl.ac.uk \\ 2 UCL Institute of Neurology, London, UK \\ p.nachev@ucl.ac.uk
}

\begin{abstract}
Automatically generating one medical imaging modality from another is known as medical image translation, and has numerous interesting applications. This paper presents an interpretable generative modelling approach to medical image translation. By allowing a common model for group-wise normalisation and segmentation of brain scans to handle missing data, the model allows for predicting entirely missing modalities from one, or a few, MR contrasts. Furthermore, the model can be trained on a fairly small number of subjects. The proposed model is validated on three clinically relevant scenarios. Results appear promising and show that a principled, probabilistic model of the relationship between multi-channel signal intensities can be used to infer missing modalities - both MR contrasts and CT images.
\end{abstract}

\section{Introduction}

This paper concerns a relatively simple method of synthesising data of one medical image modality, from data of other modalities. This is known as 'image translation'. Applications of medical image translation are numerous, and include e.g. harmonising data across scanners; synthesising computed tomography (CT) images from magnetic resonance (MR) images for positron emission tomography (PET) attenuation correction [1, or decrease the need for radiating a patient; simplifying the problem of multi-modal image registration [2]; or generalising machine learning techniques by transferring out-of-distribution input data to the domain of the model's training data 3 .

Mapping from the signal intensities of one modality to those of another can be loosely categorised as either optimisation- or learning-based. Optimisationbased methods rely only on the data at hand to optimise a mapping between modalities, and do not use training data. Examples include using non-parametric joint histograms [4, estimating an intensity transformation during image registration [5], and biophysical models [6]. Learning-based methods use training data to learn the mapping, and can be applied to translating an unseen image from one domain into another. Some examples in this category use clustering [7, random forests [8], patch-matching [9] and dictionaries [10]. Learning-based 
methods based on various convolutional neural network architectures are currently the most popular approach for this. Trained end-to-end, on either paired or unpaired training data [1112 13, they show promising results at this task, although they can run the risk of hallucinating unwanted features [14.

This paper presents a more interpretable generative modelling approach to image translation. It could be classed as an optimisation-based approach, although it does use training data to learn priors that inform the optimisation of mappings. More specifically, we show how a generative model for group-wise normalisation and segmentation of neuroimaging data can be extended to handle missing data. The generative model has a Gaussian mixture model component, which can naturally handle missing data 15 . In this paper, we extend this missing data model to a variational Gaussian mixture. Fitting this model to various populations of medical images allows us to predict, from a few MR contrasts, entirely missing modalities (e.g., non-acquired MR contrasts or CT images).

\section{Methods}

The prediction of one modality from another is here cast as a joint intensity modelling problem. The workhorse of the proposed method is the unified segmentation model [16], which uses mixtures of Gaussians with non-stationary tissue priors derived from a deformable template. When a large dataset is available, the optimisation of the template can be interleaved with the mixture model fit to each individual subject [17. Furthermore, priors over the intensity parameters of the Gaussian mixture - its means and covariances - can be defined and optimised as well. This type of learning, where subject-specific parameters are marginalised while population parameters are optimised, is known as parametric empirical Bayesian methods [18. Here, exact marginalisation is intractable, so we resort to a variational approximation.

Fully observed model: Let $\boldsymbol{X} \in \mathbb{R}^{D \times M}$ be a multimodal dataset from one subject, where $M$ is the number of modalities and $D$ is the number of voxels in the images. Each voxel is assumed to belong to one of $K$ classes, where the classification is encoded by the label matrix $\boldsymbol{Z} \in[0,1]^{D \times K}$, with $z_{d k}=1$ iff. voxel $d$ belongs to class $k$. Each tissue class is associated with a multivariate Gaussian distribution of dimension $M$, which encodes the intensities' mean $\left(\boldsymbol{\mu}_{k} \in \mathbb{R}^{M}\right)$ and covariance $\left(\boldsymbol{\Sigma}_{k} \in \mathbb{R}^{M \times M}\right)$ over the modalities. The Gaussian mixture model can then be written as a conditional probability that factorises across voxels:

$$
p\left(\boldsymbol{X} \mid \boldsymbol{Z}, \boldsymbol{\mu}_{1 \ldots K}, \boldsymbol{\Sigma}_{1 \ldots K}\right)=\prod_{d=1}^{D} \prod_{k=1}^{K} \mathcal{N}\left(\boldsymbol{x}_{d} \mid \boldsymbol{\mu}_{k}, \boldsymbol{\Sigma}_{k}\right)^{z_{d k}} .
$$

Subject-specific parameters (the label matrix and Gaussian parameters) are assumed to be drawn from prior distributions that describe their variability at the population level. Labels are drawn from a categorical distribution whose probabilities are encoded by a deformable template $\boldsymbol{a} \in \mathbb{R}^{D_{a} \times K}$. This template 
is mapped to the subject's brain using a non-linear deformation field $\phi$. This assumption can be written as the conditional likelihood:

$$
p(\boldsymbol{Z})=\prod_{d=1}^{D} \operatorname{Cat}\left(\boldsymbol{z}_{d} \mid \boldsymbol{\pi}_{d}\right), \quad \boldsymbol{\pi}_{d} \in \mathbb{R}^{K}, \quad \pi_{d k}=\frac{\exp \left(\omega_{k}+\boldsymbol{a}_{d k}(\boldsymbol{\phi})\right)}{\sum_{j=1}^{K} \exp \left(\omega_{j}+\boldsymbol{a}_{d j}(\boldsymbol{\phi})\right)},
$$

where $\boldsymbol{\omega} \in \mathbb{R}^{K}$ is a vector of global class proportions, which can be optimised to account for variable amounts of different classes (an example when modelling brain images could be atrophy due to ageing). The Gaussian parameters are drawn from their conjugate Gauss-Wishart distribution:

$$
\begin{aligned}
p\left(\boldsymbol{\mu}_{k}, \boldsymbol{\Sigma}_{k}^{-1}\right) & =\mathcal{N} \mathcal{W}\left(\boldsymbol{\mu}_{k}, \boldsymbol{\Sigma}_{k}^{-1} \mid \boldsymbol{\mu}_{0 k}, b_{0 k}, \boldsymbol{V}_{0 k}, \nu_{0 k}\right) \\
& =\mathcal{N}\left(\boldsymbol{\mu}_{k} \mid \boldsymbol{\mu}_{0 k}, \boldsymbol{\Sigma}_{k} / b_{0 k}\right) \mathcal{W}\left(\boldsymbol{\Sigma}_{k}^{-1} \mid \boldsymbol{V}_{0 k}, \nu_{0 k}\right) .
\end{aligned}
$$

Assuming that all population parameters are fixed, a mean-field approximation is made so that the posterior distribution over all latent, subject-specific parameters factorises as:

$$
q\left(\boldsymbol{Z}, \boldsymbol{\mu}_{1 \ldots K}, \boldsymbol{\Sigma}_{1 \ldots K}^{-1}\right)=\left[\prod_{d=1}^{D} q\left(\boldsymbol{z}_{d}\right)\right]\left[\prod_{k=1}^{K} q\left(\boldsymbol{\mu}_{k}, \boldsymbol{\Sigma}_{k}^{-1}\right)\right],
$$

with $q\left(\boldsymbol{z}_{d}\right)=\operatorname{Cat}\left(\boldsymbol{z}_{d} \mid \tilde{\boldsymbol{z}}_{d}\right)$ and $q\left(\boldsymbol{\mu}_{k}, \boldsymbol{\Sigma}_{k}^{-1}\right)=\mathcal{N} \mathcal{W}\left(\boldsymbol{\mu}_{k}, \boldsymbol{\Sigma}_{k}^{-1} \mid \tilde{\boldsymbol{\mu}}_{k}, \tilde{b}_{k}, \tilde{\boldsymbol{V}}_{k}, \tilde{\nu}_{k}\right)$. The posterior parameters (denoted by a tilde) can be optimised in turn by maximising the evidence lower bound (ELBO):

$$
\begin{aligned}
\mathcal{L}= & \mathbb{E}\left[\ln p\left(\boldsymbol{X} \mid \boldsymbol{Z}, \boldsymbol{\mu}_{1 \ldots K}, \boldsymbol{\Sigma}_{1 \ldots K}\right)\right] \\
& -\sum_{d=1}^{D} \mathrm{D}_{\mathrm{KL}}\left(q_{\boldsymbol{z}_{d}} \| p_{\boldsymbol{z}_{d}}\right)-\sum_{k=1}^{K} \mathrm{D}_{\mathrm{KL}}\left(q_{\boldsymbol{\mu}_{k}, \boldsymbol{\Sigma}_{k}} \| p_{\boldsymbol{\mu}_{k}, \boldsymbol{\Sigma}_{k}}\right) .
\end{aligned}
$$

When multiple subjects $\left\{\boldsymbol{X}_{n}\right\}_{n=1}^{N}$ are processed, the posterior distribution factorises across subjects and a combined ELBO can be written by summing the individual ELBOs $\left(\mathcal{L}=\sum_{n=1}^{N} \mathcal{L}_{n}\right)$. In this case, empirical population priors can be obtained by optimising the combined ELBO with respect to the template $(\boldsymbol{a})$ and Gauss-Wishart prior hyper-parameters $\left(\boldsymbol{\mu}_{0 k}, b_{0 k}, \boldsymbol{V}_{0 k}, \nu_{0 k}\right)$. The means and scale matrices have closed form solutions, while the template and degrees of freedom must be optimised using an iterative scheme. Population prior parameters and subject posterior parameters can be optimised in turn, resulting in a variational Expectation-Maximisation (VEM) algorithm [19].

Missing modalities: Let us assume that some modalities are missing in a voxe ${ }^{3}$ We write as $\boldsymbol{o}$ the vector indexing observed modalities and as $\boldsymbol{m}$ the

\footnotetext{
${ }^{3}$ For example, a multi-channel MRI might have three contrasts: T1w, T2w and PDw. In one voxel, only the $\mathrm{T} 1 \mathrm{w}$ intensity is observed. The $\mathrm{T} 2 \mathrm{w}$ and $\mathrm{PDw}$ intensities are then assumed missing in that voxel. Note that different voxels can have different combinations of contrasts/modalities missing.
} 
vector indexing missing modalities. Therefore, the observed channels can be written as $\boldsymbol{g}=\boldsymbol{x}_{\boldsymbol{o}}$ and the missing channels as $\boldsymbol{h}=\boldsymbol{x}_{\boldsymbol{m}}$, where the voxel index $d$ has been temporarily dropped for clarity. For a voxel in class $k$, the marginal distribution of the observed channels can then be written as [20]:

$$
p\left(\boldsymbol{g} \mid \boldsymbol{\mu}_{k}, \boldsymbol{\Sigma}_{k}, z_{k}=1\right)=\mathcal{N}\left(\boldsymbol{g} \mid \boldsymbol{\mu}_{k \boldsymbol{o}}, \boldsymbol{\Sigma}_{k o \boldsymbol{o}}\right),
$$

and the conditional distribution of the missing channels as:

$$
\begin{aligned}
& p\left(\boldsymbol{h} \mid \boldsymbol{g}, \boldsymbol{\mu}_{k}, \boldsymbol{\Sigma}_{k}, z_{k}=1\right)= \\
& \mathcal{N}\left(\boldsymbol{h} \mid \boldsymbol{\mu}_{k \boldsymbol{m}}-\left(\boldsymbol{\Lambda}_{k \boldsymbol{m} \boldsymbol{m}}\right)^{-1} \boldsymbol{\Lambda}_{k \boldsymbol{m} \boldsymbol{o}}\left(\boldsymbol{g}-\boldsymbol{\mu}_{k \boldsymbol{o}}\right),\left(\boldsymbol{\Lambda}_{k \boldsymbol{m} \boldsymbol{m}}\right)^{-1}\right),
\end{aligned}
$$

where the precision matrix $\boldsymbol{\Lambda}=\boldsymbol{\Sigma}^{-1}$ is the inverse of the covariance matrix.

The set of all missing values in an image is written as $\mathcal{H}=\left\{\boldsymbol{h}_{d}\right\}_{d=1}^{D}$. The mean field approximation becomes:

$$
q\left(\mathcal{H}, \boldsymbol{Z}, \boldsymbol{\mu}_{1 \ldots K}, \boldsymbol{\Sigma}_{1 \ldots K}^{-1}\right)=\left[\prod_{d=1}^{D} q\left(\boldsymbol{h}_{d} \mid \boldsymbol{z}_{d}\right) q\left(\boldsymbol{z}_{d}\right)\right]\left[\prod_{k=1}^{K} q\left(\boldsymbol{\mu}_{k}, \boldsymbol{\Sigma}_{k}^{-1}\right)\right],
$$

where $q\left(\boldsymbol{h}_{d} \mid \boldsymbol{z}_{d}\right)=\prod_{k=1}^{K} \mathcal{N}\left(\boldsymbol{h}_{d} \mid \tilde{\boldsymbol{h}}_{d k}, \tilde{\boldsymbol{S}}_{d k}\right)^{z_{d k}}$. The marginal posterior over missing values is a mixture of Gaussians that can be obtained by marginalising the labels:

$$
q\left(\boldsymbol{h}_{d}\right)=\sum_{k=1}^{K} \tilde{z}_{d k} \mathcal{N}\left(\boldsymbol{h}_{d} \mid \tilde{\boldsymbol{h}}_{d k}, \tilde{\boldsymbol{S}}_{d k}\right) .
$$

Its expected value is $\mathbb{E}\left[\boldsymbol{h}_{d}\right]=\sum_{k} \tilde{z}_{d k} \tilde{\boldsymbol{h}}_{d k}$. This is the expression that we evaluate to predict missing voxels.

The set of all observed values is written as $\mathcal{G}=\left\{\boldsymbol{g}_{d}\right\}_{d=1}^{D}$. The ELBO can then be written in two equivalent forms:

$$
\begin{aligned}
\mathcal{L}= & \mathbb{E}\left[\ln p\left(\mathcal{G} \mid \boldsymbol{Z}, \boldsymbol{\mu}_{1 \ldots K}, \boldsymbol{\Sigma}_{1 \ldots K}\right)\right] \\
& -\sum_{d=1}^{D} \mathrm{D}_{\mathrm{KL}}\left(q_{\boldsymbol{z}_{d}} \| p_{\boldsymbol{z}_{d}}\right)-\sum_{k=1}^{K} \mathrm{D}_{\mathrm{KL}}\left(q_{\boldsymbol{\mu}_{k}, \boldsymbol{\Sigma}_{k}} \| p_{\boldsymbol{\mu}_{k}, \boldsymbol{\Sigma}_{k}}\right) \\
\mathcal{L}= & \mathbb{E}\left[\ln p\left(\boldsymbol{X} \mid \boldsymbol{Z}, \boldsymbol{\mu}_{1 \ldots K}, \boldsymbol{\Sigma}_{1 \ldots K}\right)\right]-\sum_{d=1}^{D} \mathbb{E}_{\boldsymbol{z}_{d}}\left[\mathrm{D}_{\mathrm{KL}}\left(q_{\boldsymbol{h}_{d} \mid \boldsymbol{z}_{d}} \| p_{\boldsymbol{h}_{d} \mid \boldsymbol{z}_{d}}\right)\right] \\
& -\sum_{d=1}^{D} \mathrm{D}_{\mathrm{KL}}\left(q_{\boldsymbol{z}_{d}} \| p_{\boldsymbol{z}_{d}}\right)-\sum_{k=1}^{K} \mathrm{D}_{\mathrm{KL}}\left(q_{\boldsymbol{\mu}_{k}, \boldsymbol{\Sigma}_{k}} \| p_{\boldsymbol{\mu}_{k}, \boldsymbol{\Sigma}_{k}}\right) .
\end{aligned}
$$

The first form is used to optimise the labels' posterior parameters, while the second is used to optimise, in turn, the missing values and the Gaussian posterior parameters. 
Model updates: Optimising the ELBOs in (10) and (11) gives the subject-level update equations as:

$$
\begin{aligned}
\tilde{z}_{d k} & =\frac{\exp \left(\mathbb{E}\left[\ln \mathcal{N}\left(\boldsymbol{g}_{d} \mid \boldsymbol{\mu}_{k}, \boldsymbol{\Sigma}_{k}\right)\right]+\ln \pi_{d k}\right)}{\sum_{l=1}^{K} \exp \left(\mathbb{E}\left[\ln \mathcal{N}\left(\boldsymbol{g}_{d} \mid \boldsymbol{\mu}_{l}, \boldsymbol{\Sigma}_{l}\right)\right]+\ln \pi_{d l}\right)} \\
\tilde{b}_{k} & =b_{0 k}+\sum_{d=1}^{D} \tilde{z}_{d k} \\
\tilde{\boldsymbol{\mu}}_{k} & =\frac{b_{0 k} \boldsymbol{\mu}_{0 k}+\sum_{d=1}^{D} \mathbb{E}\left[z_{d k} \boldsymbol{x}_{d}\right]}{\tilde{b}_{k}} \\
\tilde{\nu}_{k} & =\nu_{0 k}+\sum_{d=1}^{D} \tilde{z}_{d k} \\
\tilde{\boldsymbol{V}}_{k}^{-1} & =\nu_{0 k} \boldsymbol{V}_{0 k}^{-1}+\sum_{d=1}^{D} \mathbb{E}\left[z_{d k} \boldsymbol{x}_{d} \boldsymbol{x}_{d}^{\mathrm{T}}\right]+b_{k 0} \boldsymbol{\mu}_{k 0} \boldsymbol{\mu}_{k 0}^{\mathrm{T}}-\tilde{b}_{k} \tilde{\boldsymbol{\mu}}_{k} \tilde{\boldsymbol{\mu}}_{k}^{\mathrm{T}}
\end{aligned}
$$

The update equations for the Gaussian parameters in the missing data case are very similar to the fully observed case, except that expectations are taken about the data. These expectations are evaluated as:

$$
\begin{aligned}
& \mathbb{E}\left[z_{d k} \boldsymbol{x}_{d}\right]_{\boldsymbol{o}}=\tilde{z}_{d k} \boldsymbol{g}_{d}, \quad \mathbb{E}\left[z_{d k} \boldsymbol{x}_{d} \boldsymbol{x}_{d}^{\mathrm{T}}\right]_{\boldsymbol{m m}}=\tilde{z}_{d k}\left(\tilde{\boldsymbol{h}}_{d k} \tilde{\boldsymbol{h}}_{d k}^{\mathrm{T}}+\tilde{\boldsymbol{S}}_{d k}\right), \\
& \mathbb{E}\left[z_{d k} \boldsymbol{x}_{d}\right]_{\boldsymbol{m}}=\tilde{z}_{d k} \tilde{\boldsymbol{h}}_{d k}, \quad \mathbb{E}\left[z_{d k} \boldsymbol{x}_{d} \boldsymbol{x}_{d}^{\mathrm{T}}\right]_{\text {om }}=\tilde{z}_{d k} \boldsymbol{g}_{d} \tilde{\boldsymbol{h}}_{d k}^{\mathrm{T}}, \\
& \mathbb{E}\left[z_{d k} \boldsymbol{x}_{d} \boldsymbol{x}_{d}^{\mathrm{T}}\right]_{\boldsymbol{o o}}=\tilde{z}_{d k} \boldsymbol{g}_{d} \boldsymbol{g}_{d}^{\mathrm{T}}, \quad \mathbb{E}\left[z_{d k} \boldsymbol{x}_{d} \boldsymbol{x}_{d}^{\mathrm{T}}\right]_{\boldsymbol{m o}}=\tilde{z}_{d k} \tilde{\boldsymbol{h}}_{d k} \boldsymbol{g}_{d}^{\mathrm{T}},
\end{aligned}
$$

where

$$
\tilde{\boldsymbol{h}}_{d k}=\tilde{\boldsymbol{\mu}}_{k \boldsymbol{m}}-\tilde{\boldsymbol{\Lambda}}_{k \boldsymbol{m} \boldsymbol{m}}^{-1} \tilde{\boldsymbol{\Lambda}}_{k \boldsymbol{m} \boldsymbol{o}}\left(\boldsymbol{g}_{d}-\tilde{\boldsymbol{\mu}}_{k \boldsymbol{o}}\right), \quad \tilde{\boldsymbol{S}}_{d k}=\tilde{\boldsymbol{\Lambda}}_{k \boldsymbol{m} \boldsymbol{m}}^{-1}
$$

and $\tilde{\boldsymbol{\Lambda}}_{k}=\tilde{\nu}_{k} \tilde{\boldsymbol{V}}_{k}$ is the posterior expected precision matrix of a given class.

Finally, we provide the optimal updates of the Gaussian prior parameters, given a set of individual posterior parameters. All prior parameters have closedform updates, except for the degrees of freedom of the Wishart distribution, which is updated using an iterative Gauss-Newton scheme. The update equations 
are:

$$
\begin{aligned}
\boldsymbol{\mu}_{0 k} & =\left(\sum_{n=1}^{N} \tilde{\nu}_{n k} \tilde{\boldsymbol{V}}_{n k}\right)^{-1}\left(\sum_{n=1}^{N} \tilde{\nu}_{n k} \tilde{\boldsymbol{V}}_{n k} \tilde{\boldsymbol{\mu}}_{n k}\right) \\
b_{0 k}^{-1} & =\frac{1}{N M} \sum_{n=1}^{N} \tilde{\nu}_{n k}\left(\boldsymbol{\mu}_{0 k}-\tilde{\boldsymbol{\mu}}_{n k}\right)^{\mathrm{T}} \tilde{\boldsymbol{V}}_{n k}\left(\boldsymbol{\mu}_{0 k}-\tilde{\boldsymbol{\mu}}_{n k}\right) \\
\boldsymbol{V}_{0 k} & =\frac{1}{N \nu_{0 k}} \sum_{n=1}^{N} \tilde{\nu}_{n k} \tilde{\boldsymbol{V}}_{n k} \\
\frac{\partial \mathcal{L}}{\partial \nu_{0 k}} & =-\frac{1}{2}\left(N\left(\ln \left|\boldsymbol{V}_{0 k}\right|+\psi_{M}\left(\frac{\nu_{0 k}}{2}\right)\right)-\sum_{n=1}^{N}\left(\ln \left|\tilde{\boldsymbol{V}}_{n k}\right|-\psi_{M}\left(\frac{\tilde{\nu}_{n k}}{2}\right)\right)\right) \\
\frac{\partial^{2} \mathcal{L}}{\partial \nu_{0 k}^{2}} & =-\frac{N}{4} \psi_{M}^{\prime}\left(\frac{\tilde{\nu}_{0 k}}{2}\right)
\end{aligned}
$$

We do not provide update rules for the template $(\boldsymbol{a})$, as they can be found in [17.

\section{Experiments and Results}

In this section we aim to explore the translation (or inference) capability of the proposed model by conducting three experiments on publicly available data. We investigate: (1) inferring missing voxels of MRIs with differing field of views; (2) inferring entirely missing MRI contrasts; and (3), inferring CT scans from MRIs. The findings are quantified by computing the peak-signal-to-noise-ratio (PSNR) for an image channel $c$ as:

$$
\mathrm{PSNR}=10 \log _{10} \frac{\text { maxval }^{2}}{M S E}
$$

where the mean-squared error is defined as MSE $=\frac{1}{D} \sum_{d=1}^{D}\left(\hat{x}_{c d}-\left(\mathbb{E}\left[\boldsymbol{h}_{d}\right]\right)_{c}\right)^{2}$, maxval is the maximum channel intensity in the reference image $\hat{\boldsymbol{X}}$, and $\mathbb{E}\left[\boldsymbol{h}_{d}\right]$ from (9) is evaluated to predict missing voxels. The PSNR is a metric that is commonly used in the medical image synthesis literature [11/13]12. Note that no voxels are excluded when computing the PSNR.

\subsection{MRI Contrast Translation}

This section evaluates translating between MR contrasts. The model is trained on 50 subjects from the publicly available IXI dataset 4 which was acquired on

\footnotetext{
4 http://brain-development.org/ixi-dataset/
} 

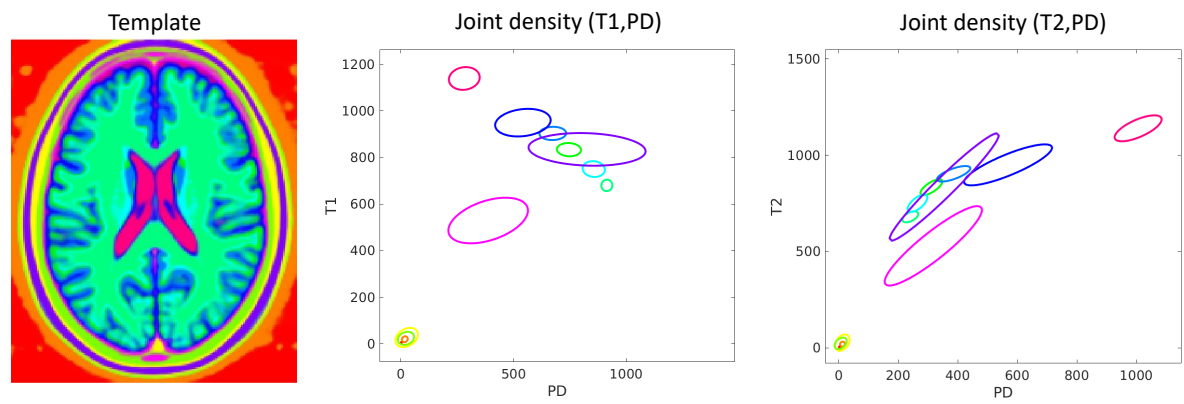

Fig. 1: Template and expectations of the Gaussians drawn from the GaussWishart priors, learnt from 50 IXI subjects. Densities are plotted using their $3 \sigma$ isocontours. This model is fit to a new subject, which allows for inferring missing voxels.

three different MR scanner:5 Each IXI subject has three MR images: a T1, T2- and PD-weighted scan (T1w, T2w and PDw). Furthermore, the images have approximately $1 \mathrm{~mm}$ isotropic voxels and all subjects are healthy. $K=12$ mixture components are used, resulting in the model shown in Fig. 1. Note that the template learned by the algorithm does not need to represent real tissues. Here, the model has been treated as a method of representing a probability density function, rather than as a way to do clustering. Any 'meaningful' clusters are incidental.

Inferring MRIs with Differing Fields of View: Doctors often acquire routine clinical MR scans of multiple contrasts. Commonly, these contrasts have differing fields of view, meaning the brain coverage varies (cf. observed T1w and T2w images in Fig. 2). This can be problematic for image segmentation routines as voxels with non-observed contrasts need to be discarded. The model should prevent this issue by inferring the values of these missing voxels. To test this, $\mathrm{T} 1 \mathrm{w}, \mathrm{T} 2 \mathrm{w}$ and PDw scans of 50 unseen IXI subjects are used ${ }^{6}$ All of the voxels are retained in the PDw image, while an increasing amount of voxels are removed from the $\mathrm{T} 1 \mathrm{w}$ and $\mathrm{T} 2 \mathrm{w}$ images $(25 \%, 50 \%, 75 \%$ and 100\%). The missing voxels are then inferred with the trained model. An example can be seen in Fig. 2. The mean PSNR computed between the known references and the inferred images are shown in Table 2, For routine clinical MRI, it is rare that more than $50 \%$ of the field of view is missing. The results therefore suggest that the model does a good job at filling in missing fields of view, which could be of value in segmenting hospital data.

\footnotetext{
${ }^{5}$ This scenario is more realistic in a clinical context. The results would improve if data from only one scanners was used.

6 The model is trained on IXI subjects IXI [064-118], and tested on IXI [002-063].
} 


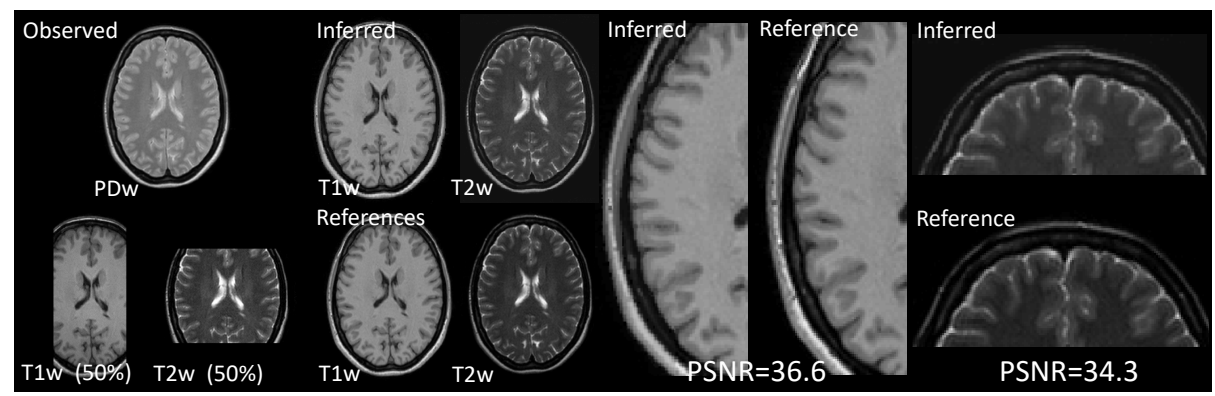

Fig. 2: Example of inferring MRIs with differing field of views. An MR image with three channels (PDw, T1w and T2w) is observed. The PDw scan has full brain coverage, while the $\mathrm{T} 1 \mathrm{w}$ and $\mathrm{T} 2 \mathrm{w}$ scans have partial brain coverage $(50 \%$ of voxels removed in each channel). From the observed data the values of the missing $\mathrm{T} 1 \mathrm{w}$ and $\mathrm{T} 2 \mathrm{w}$ voxels are inferred. The reference $\mathrm{T} 1 \mathrm{w}$ and $\mathrm{T} 2 \mathrm{w}$ scans are shown for comparison, as well as PSNR values.

Table 1: Results for inferring MR images with different fields of view (for 50 subjects). The PSNR is computed between known T2w and PDw references and inferred images, where an increasing percentage of the field of view has been removed. Results are shown as mean \pm std.

\begin{tabular}{c|cccc}
\hline & & & PSNR & \\
Contrast & $\mathbf{2 5 \%}$ & $\mathbf{5 0 \%}$ & $\mathbf{7 5 \%}$ & $\mathbf{1 0 0 \%}$ \\
\hline T1w & $42.1 \pm 1.6$ & $36.3 \pm 1.3$ & $31.1 \pm 1.3$ & $28.9 \pm 1.2$ \\
T2w & $40.7 \pm 2.1$ & $34.4 \pm 2.0$ & $30.4 \pm 1.8$ & $27.6 \pm 1.6$ \\
\hline
\end{tabular}

Inferring MR Contrasts: Could the proposed model be used to infer an entirely missing MR contrast? An interesting application for this type of MRI translation could be for segmentation methods based on deep learning. A deep learning model that has been trained on MR images of a specific contrast can overfit to its training data 21. If images could be simulated as to match the training data of the deep learning model, it might generalise better.

To test how well the model predict a missing contrast the same IXI subjects as in the previous experiment are used. For each subject, all combinations of contrasts are permuted over, set as either observed or missing. For example, we observe just the $\mathrm{T} 1 \mathrm{w}$ image and infer the $\mathrm{T} 2 \mathrm{w}$ and $\mathrm{PDw}$ scans, or we observe the T2w and PDw scans and infer the T1w (see Fig. 3). The results from this experiment are shown in Table 2 . These results imply that the $\mathrm{T} 1 \mathrm{w}$ image is the most predictive, as the lowest PSNR is obtained when this contrast is missing. The example inferred PDw image in Fig. 3 looks realistic when compared to the known reference, although more noisy. The results in Table 2 are close to those previously reported in the literature [11] (for the same task but a different dataset). 


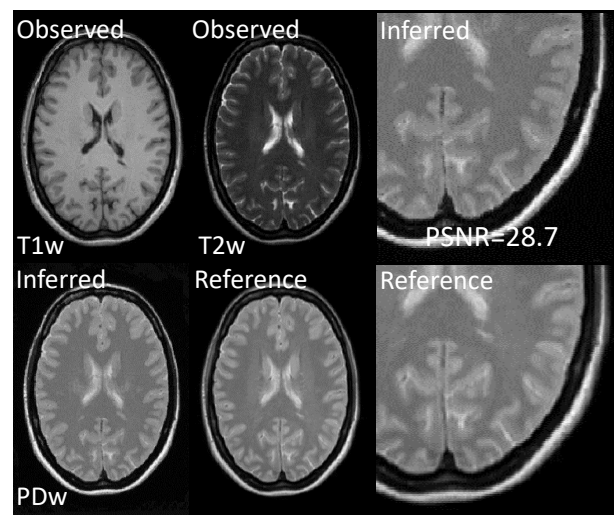

Fig. 3: Example of inferring non-acquired MR contrasts. An MR image with two channels ( $\mathrm{T} 1 \mathrm{w}$ and $\mathrm{T} 2 \mathrm{w}$ ) is observed. The PDw scan is missing, but inferred from the observed $\mathrm{T} 1 \mathrm{w}$ and $\mathrm{T} 2 \mathrm{w}$ scans. The reference PDw scan is shown for comparison, as well as the PSNR value.

Table 2: Results for inferring MR image contrasts (for 50 subjects). PSNR is computed for all different permutations of observed and missing contrasts. Results are shown as mean \pm std.

\begin{tabular}{cc|ccc}
\hline \multicolumn{2}{c}{ Contrasts } & \multicolumn{3}{c}{ PSNR } \\
Observed & Missing & T1w & T2w & PDw \\
\hline T1w & T2w, PDw & - & $28.9 \pm 1.5$ & $28.5 \pm 1.1$ \\
T2w & T1w, PDw & $28.2 \pm 1.0$ & - & $28.3 \pm 1.5$ \\
PDw & T1w, T2w & $28.0 \pm 1.2$ & $27.6 \pm 1.6$ & - \\
T1w,T2w & PDw & - & - & $28.8 \pm 0.9$ \\
T2w,PDw & T1w & $29.2 \pm 1.4$ & - & - \\
T1w,PDw & T2w & - & $28.1 \pm 1.5$ & - \\
\hline
\end{tabular}

\subsection{MRI to CT Translation}

Accurately translating MRIs to CTs is interesting for numerous reasons, e.g., for removing the exposure to radiation that $\mathrm{CT}$ imaging involves, or for attenuation correction in MR-PET imaging. The proposed model should allow for this type of translation, by training it on subjects who have both MR and CT imaging. We therefore retrain the intensity distribution hyper-parameters of the model retaining the template learnt from the IXI dataset - on eight patients from the RIRE dataset ${ }^{7}$ 22. Each patient in this dataset contains a number of imaging modalities. Here, only the patients with $\mathrm{T} 1 \mathrm{w}$ and $\mathrm{T} 2 \mathrm{w}$ MR scans (non-rectified), and CT images, are used. Note that the RIRE dataset is challenging to use due to the images having thick-slices, sometimes pathology, as well as requiring an initial co-registration (the dataset is part of a registration challenge and therefore purposefully misaligned). Each subject's scans are registered using the co-registration routine of the SPM12 software.

\footnotetext{
7 https://www .insight-journal .org/rire/
} 


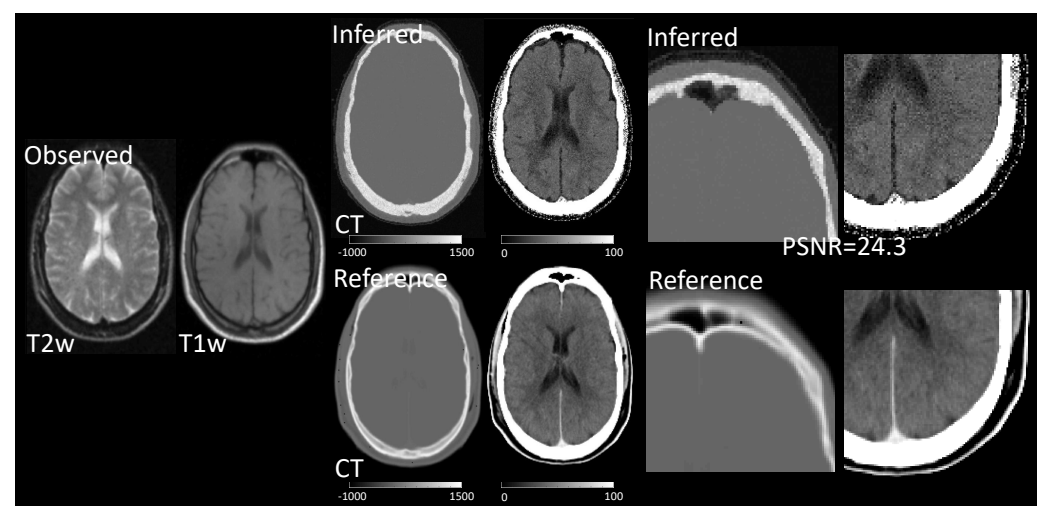

Fig. 4: Example of MRI to CT translation. An MR image with two channels (T1w and T2w) is observed. A CT scan is then inferred from the observed T1w and $\mathrm{T} 2 \mathrm{w}$ scans. The reference CT scan is shown for comparison, as well as the PSNR value.

To test the models ability to translate MRIs to CTs, eight unseen RIRE patients are used ${ }^{8}$ The trained model is fit to each subject's T1w and T2w scans. The expected marginal posterior distribution over the missing CT image can then be computed. An example is shown in Fig. 4. The mean \pm std PSNR between the inferred CT images and the known references is $25.5 \pm 1.2$. Considered the intensity hyper-parameters were trained on only eight subjects, the results are satisfactory, although not on pair with deep learning based techniques [13. The examples images in Fig. 4 suggests that the model does not capture a detailed enough distribution of bone. Additionally, the meninges does not appear in the inferred image, but is instead modelled as cerebrospinal fluid. Fitting not only the intensity hyper-parameters to the $\mathrm{CT}$ data, but also the template, could resolve these issues. More training data would also help.

\section{Conclusion}

This paper showed how a popular model for segmenting brain scans - a probabilistic forward model with a Gaussian mixture part - can be extended to infer missing data. For multi-channel segmentation, this extension circumvents the need to model only voxels that are observed in all channels. It furthermore enables predicting one MR contrast from another, or CTs from MRIs. The model gives reasonable results if trained on a small number of subjects, but we would expect further improvements with access to more training data. Interestingly, image translation is just a 'by-product' of learning the parameters of a joint

\footnotetext{
8 The model is trained on RIRE patients patient[102-109], and tested on patient $[001-007,101]$.
} 
probability distribution that models missing voxels. The same model can also be used to segment, bias correct and spatially normalise brain scans.

The model requires setting the number of Gaussian mixture components $(K)$ at the start of the training. If this number is set too low, then the simulated images will look unrealistic. Here, this issue was resolved by using a fairly large number of components, which was found empirically capturing a detailed enough model distribution. Uninformative mixture components can then be drived to zero, due to the Bayesian setting of the Gaussian mixture model, by making point estimates of the values of the global tissue proportions $(\boldsymbol{\omega})$. This is known as automatic relevance determination [20].

Generative modelling approaches integrating multi-channel images, like the one presented here, should involve a component that relates signal across the various channels. The approach presented in this paper involves a probabilistic model of the relationship between signal intensities over channels. An alternative approach would be to use a multi-channel total variation (MTV) prior, which ensures that 'edges' appear in similar locations across channels. The MTV prior can be used to achieve super-resolution or denoising of medical images [23]. An avenue of future work could therefore be to incorporate both of these components into a super-resolution method, to improve resolution of thick-sliced, hospitalgrade MR scans. By combining, for example, axial thick-sliced T2-weighted images and sagittal thick-sliced T1w images of the same subjects. In this example, the $\mathrm{T} 2 \mathrm{w}$ image could provide some of the missing $\mathrm{T} 1 \mathrm{w}$ signal in the left-right direction, whereas the $\mathrm{T} 1 \mathrm{w}$ image could fill in some of the missing $\mathrm{T} 2 \mathrm{w}$ signal in the inferior-posterior direction. Of course, this strategy would need to be formulated properly, but this work aimed to show a proof of the concept that one of those components, a probabilistic model between channels, does a good job at filling in missing data in MR images.

Acknowledgements: MB was funded by the EPSRC-funded UCL Centre for Doctoral Training in Medical Imaging (EP/L016478/1) and the Department of Healths NIHR-funded Biomedical Research Centre at University College London Hospitals. MB and JA was funded by the EU Human Brain Project's Grant Agreement No 785907 (SGA2). YB was funded by the MRC and Spinal Research Charity through the ERA-NET Neuron joint call (MR/R000050/1).

\section{References}

1. N. Burgos, M. J. Cardoso, M. Modat, S. Pedemonte, J. Dickson, A. Barnes, J. S. Duncan, D. Atkinson, S. R. Arridge, B. F. Hutton, et al., "Attenuation correction synthesis for hybrid PET-MR scanners," in MICCAI, pp. 147-154, Springer, 2013.

2. T. Cao, C. Zach, S. Modla, D. Powell, K. Czymmek, and M. Niethammer, "Registration for correlative microscopy using image analogies," in WBIR, pp. 296-306, Springer, 2012.

3. S. Roy, A. Carass, N. Shiee, D. L. Pham, and J. L. Prince, "MR contrast synthesis for lesion segmentation," in ISBI, pp. 932-935, IEEE, 2010. 
4. D.-J. Kroon and C. H. Slump, "MRI modalitiy transformation in demon registration," in ISBI, pp. 963-966, IEEE, 2009.

5. A. Guimond, A. Roche, N. Ayache, and J. Meunier, "Three-dimensional multimodal brain warping using the demons algorithm and adaptive intensity corrections," IEEE T. Med. Imaging, vol. 20, no. 1, pp. 58-69, 2001.

6. W. Wein, S. Brunke, A. Khamene, M. R. Callstrom, and N. Navab, "Automatic CT-ultrasound registration for diagnostic imaging and image-guided intervention," Med. Image Anal., vol. 12, no. 5, pp. 577-585, 2008.

7. S.-H. Hsu, Y. Cao, K. Huang, M. Feng, and J. M. Balter, "Investigation of a method for generating synthetic CT models from MRI scans of the head and neck for radiation therapy," Phys. Med. Biol., vol. 58, no. 23, p. 8419, 2013.

8. T. Huynh, Y. Gao, J. Kang, L. Wang, P. Zhang, J. Lian, and D. Shen, "Estimating CT image from MRI data using structured random forest and auto-context model," IEEE T. Med. Imaging, vol. 35, no. 1, pp. 174-183, 2015.

9. J. E. Iglesias, E. Konukoglu, D. Zikic, B. Glocker, K. Van Leemput, and B. Fischl, "Is synthesizing MRI contrast useful for inter-modality analysis?," in MICCAI, pp. 631-638, Springer, 2013.

10. S. Roy, A. Carass, and J. Prince, "A compressed sensing approach for MR tissue contrast synthesis," in IPMI, pp. 371-383, Springer, 2011.

11. A. Chartsias, T. Joyce, M. V. Giuffrida, and S. A. Tsaftaris, "Multimodal mr synthesis via modality-invariant latent representation," IEEE transactions on medical imaging, vol. 37, no. 3, pp. 803-814, 2017.

12. D. Nie, R. Trullo, J. Lian, C. Petitjean, and M. Ruan, "Medical image synthesis with context-aware generative adversarial networks," pp. 417-425, Springer, 2017.

13. J. M. Wolterink, A. M. Dinkla, M. H. Savenije, P. R. Seevinck, C. A. van den Berg, and I. Išgum, "Deep MR to CT synthesis using unpaired data," in SASHIMI, pp. 14-23, Springer, 2017.

14. J. P. Cohen, M. Luck, and S. Honari, "Distribution matching losses can hallucinate features in medical image translation," in MICCAI, pp. 529-536, Springer, 2018.

15. Z. Ghahramani and M. I. Jordan, "Supervised learning from incomplete data via an EM approach," in NeurIPS, pp. 120-127, 1994.

16. J. Ashburner and K. J. Friston, "Unified segmentation," NeuroImage, vol. 26, no. 3, pp. 839-851, 2005.

17. C. Blaiotta, P. Freund, M. J. Cardoso, and J. Ashburner, "Generative diffeomorphic modelling of large MRI data sets for probabilistic template construction," NeuroImage, vol. 166, pp. 117-134, 2018.

18. B. P. Carlin and T. A. Louis, "Empirical Bayes: Past, present and future," J. Am. Stat. Assoc., vol. 95, no. 452, pp. 1286-1289, 2000.

19. C. Blaiotta, M. J. Cardoso, and J. Ashburner, "Variational inference for medical image segmentation," Comput. Vis. Image Und., vol. 151, pp. 14-28, 2016.

20. C. M. Bishop, Pattern recognition and machine learning. Springer, 2006.

21. M. Brudfors, Y. Balbastre, and J. Ashburner, "Nonlinear markov random fields learned via backpropagation," in IPMI, pp. 805-817, Springer, 2019.

22. J. B. West, ..., and R. P. Woods, "Comparison and evaluation of retrospective intermodality image registration techniques," in Medical Imaging 1996: Image Processing, vol. 2710, pp. 332-348, SPIE, 1996.

23. M. Brudfors, Y. Balbastre, P. Nachev, and J. Ashburner, "MRI super-resolution using multi-channel total variation," in MIUA, pp. 217-228, Springer, 2018. 